\title{
Designing synthetic optical media: photonic crystals
}

\author{
Steven G. Johnson, J.D. Joannopoulos * \\ Department of Physics and Center for Materials Science and Engineering, Room 12-116, Massachusetts Institute of Technology, \\ Cambridge, MA 02139-4307, USA
}

Accepted 31 August 2003

\begin{abstract}
A new class of materials, called photonic crystals, affect a photon's properties in much the same way that a semiconductor affects an electron's properties. This represents an ability to mold and guide light that leads naturally to novel applications in several fields, including optoelectronics and telecommunications. We present an introductory survey of the basic concepts and ideas that underlie photonic crystals, and present results and devices that illustrate their potential to circumvent limits of traditional optical systems.
\end{abstract}

(C) 2003 Acta Materialia Inc. Published by Elsevier Ltd. All rights reserved.

Keywords: Photonic crystals; Band gaps; Optical devices

\section{Introduction}

Over the last half-century, perhaps no basic scientific development has so affected our lives as the maturation of semiconductor physics and the transistor electronics it enabled. The key to this technology was the ability to design crystalline materials that tailor the conduction properties of electrons to suit diverse applications. The optical communications revolution, however, has shifted the frontier in high-speed, wide-bandwidth infor-

* Corresponding author. Tel.: +1-617-253-4806; fax: +1617-253-2562.

E-mail address: joannop@mit.edu (J.D. Joannopoulos).

The Golden Jubilee Issue-Selected topics in Materials Science and Engineering: Past, Present and Future, edited by S. Suresh. mation processing from electrons to photons, a shift that demands a new kind of synthetic material to mold the gigabits. To address this need, recent research has suggested a way to tailor the propagation of light much like that of electrons, via the creation of periodic optical media dubbed photonic crystals $[1,2]$. These crystals, stemming from the pioneering work of Yablonovitch and John [3,4], consists of a periodic array of macroscopic (wavelength-scale) dielectric and/or metallic "atoms" through which light passes, altered but still propagating, just as electrons filter without scattering through an array of ions in a perfect crystalline conductor. The photons are described by a band structure, via the traditional mathematics of solid-state physics, and of particular interest is a photonic crystal whose band structure displays a complete photonic band gap (PBG): a range of 
frequencies for which light cannot propagate in the crystal. In this range, such a crystal forms a kind of optical insulator, into which localized states can be introduced by the creation of intentional defects in the crystal, like dopant atoms in a semiconductor. Unlike atomic systems, however, at the photon's micrometer scale, our control over the size, shape, and symmetry of these defects is, in principle, virtually unlimited. Correspondingly, defect states can be tuned to lie at any frequency and cover any spatial extent; morever, one also has control over the symmetry of the localized photon state. All these capabilities contribute new dimensions to our ability to mold the properties of light. In this sense, defects in photonic crystals are good things: they promise the ability to manipulate light in ways that may not have been possible before. In this article, we will outline the theoretical framework for understanding photonic crystals, and highlight some of the novel possibilities they introduce.

\section{Band diagrams and gaps}

The study of wave propagation in three-dimensionally periodic media was pioneered by Felix Bloch in 1928 [5], extending an 1883 theorem in one dimension by Floquet [6]. Bloch proved that waves in such a medium can propagate without scattering, their behavior governed by a periodic envelope function multiplied by a planewave [7]. Although Bloch studied quantum mechanics, leading to the surprising result that electrons in a conductor scatter only from imperfections and not from the periodic ions, the same techniques can be applied to electromagnetism by casting Maxwell's equations as an eigenproblem in analogue with Schrödinger's equation. By combining the sourcefree Faraday's and Ampere's laws at a fixed frequency $\omega\left(\right.$ time dependence $\mathrm{e}^{-\mathrm{i} \omega t}$ ), one can obtain an equation in only the magnetic field $\mathbf{H}$ :

$$
\nabla \times \frac{1}{\varepsilon} \nabla \times \mathbf{H}=\left(\frac{\omega}{c}\right)^{2} \mathbf{H},
$$

where $\varepsilon$ is the dielectric function $\varepsilon(\mathbf{x})$ and $c$ is the speed of light. This is an eigenvalue equation, with eigenvalue $(\omega / c)^{2}$ and an eigenoperator $\nabla \times \frac{1}{\varepsilon} \nabla \times$ that is Hermitian (acts the same to the left and right) under the inner product $\int \mathbf{H}^{*} \cdot \mathbf{H}^{\prime}$ between two fields $\mathbf{H}$ and $\mathbf{H}^{\prime}$. The two curls correspond roughly to the "kinetic energy", and $1 / \varepsilon$ to the "potential", compared to the Schrödinger Hamiltonian $\nabla^{2}+V(\mathbf{x})$. An important difference compared to quantum mechanics is that there is a transversality constraint: one typically excludes $\nabla \cdot \mathbf{H} \neq 0$ (or $\nabla \cdot \varepsilon \mathbf{E}$ $\neq 0$ ) eigensolutions, which lie at $\omega=0$; i.e. staticfield solutions with free magnetic (or electric) charge are forbidden.

Thus, the same linear-algebraic theorems as those in quantum mechanics can be applied to the electromagnetic wave solutions. The fact that the eigenoperator is Hermitian and positive-definite (for real $\varepsilon>0$ ) implies that the eigenfrequencies $\omega$ are real, for example, and also leads to orthogonality, variational, and perturbation-theory relations. Most importantly for photonic crystals, however, it means that for the case of $\varepsilon$ with spatial periodicity, Bloch's theorem applies: the magnetic field can be chosen of the form $\mathbf{H}(\mathbf{x})=$ $\mathrm{e}^{\mathrm{i} \mathbf{k} \cdot \mathbf{x}} \mathbf{H}_{n, \mathbf{k}}(\mathbf{x})$ with eigenvalues $\omega_{n}(\mathbf{k})$, where $\mathbf{k}$ is the Bloch wavevector and $\mathbf{H}_{n, \mathbf{k}}$ is a periodic envelope function satisfying an eigenproblem similar to (1).

For each wavevector $\mathbf{k}$, one computes the discrete eigenvalues $\omega_{n}$, which when plotted vs. wavevector form the familiar band diagrams from solid-state physics. A corollary of Bloch's theorem states that these solutions (bands) are periodic in $\mathbf{k}$, so one need only consider a finite region of $\mathbf{k}$ space called the irreducible Brillouin zone, and in many cases the interesting behavior occurs only on the boundaries of this zone.

For comparison, in Fig. 1, we show the band structures of analogous electronic and photonic crystals, in both cases plotted along important high-symmetry directions for the Brillouin zone of an fcc/diamond lattice. In Fig. 1 (left) is the band structure of the valence electrons for silicon atoms (which naturally form a diamond structure with a lattice constant of a few angstroms), which has a band gap (a range of energies in which there are no electronic states) that creates its semiconductor properties. In Fig. 1 (right) is the band structure 
Silicon Crystal

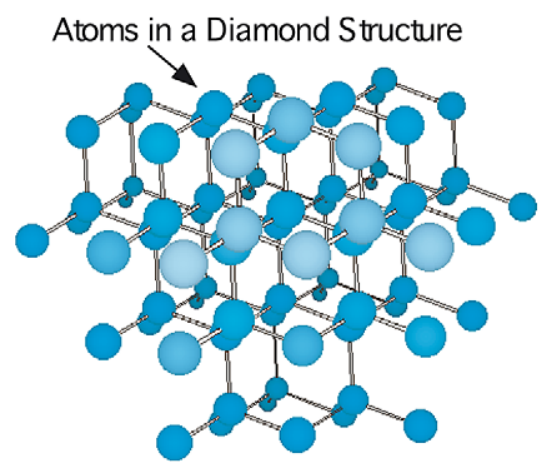

Electronic Bandstructure

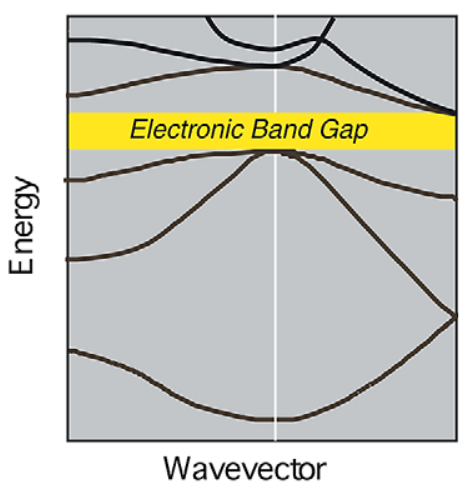

3D Photonic Crystal

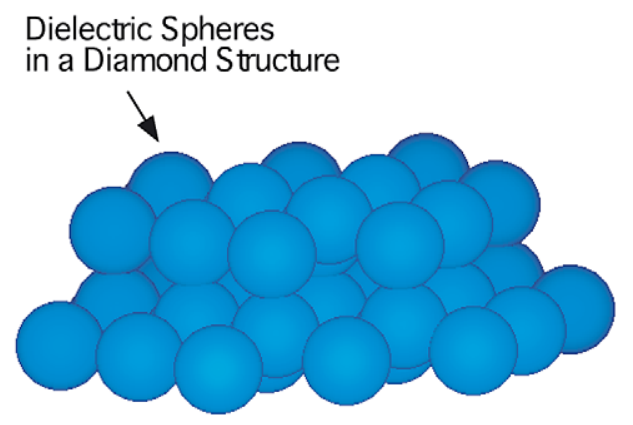

Photonic Bandstructure

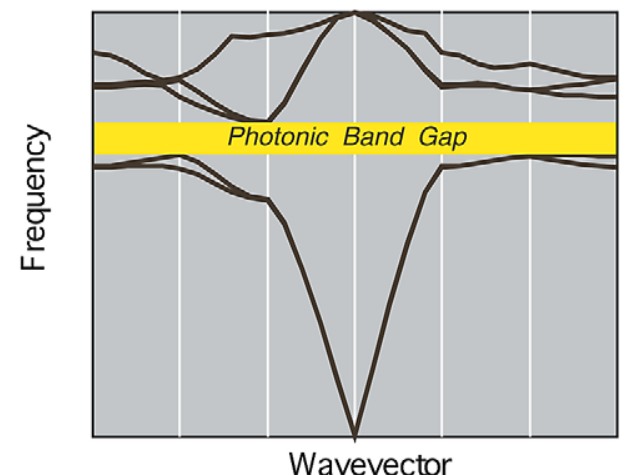

Fig. 1. Comparison of the band structures of (left) valence electrons in the diamond structure of atomic silicon and (right) photons in a synthetic diamond structure of overlapping dielectric spheres (with $\varepsilon=12$, e.g. silicon at $1.55 \mu \mathrm{m}$ ). The band gap, the range of energies/frequencies in which electrons/photons cannot propagate, is highlighted in yellow.

for a diamond lattice of overlapping dielectric (e.g. silicon) spheres in air [8], which has a maximum photonic band gap of about $11 \%$ (gap/midgap) for silicon-like $\varepsilon$, here with a lattice constant of about half of the midgap wavelength, whatever that wavelength is chosen to be-Maxwell's equations, unlike quantum mechanics, are scale-invariant and the same solutions apply at any lengthscale. Also, unlike quantum mechanics, the lowest bands drop all the way to $\omega=0$ at $\mathbf{k}=0$ in a characteristic $\omega \sim|\mathbf{k}|$ shape, corresponding to constant-field solutions; this is a consequence of the fact that the $\varepsilon$ "potential" is mixed up with the $\nabla \times$ "kinetic energy", rather than a separate additive term.

From a theoretical perspective, photonic crystals possess a considerable advantage over their elec- tronic counterparts, in that they can be simulated efficiently to arbitrary accuracy on a computer, without the draconian approximations forced by the entanglements and strong interactions of fermionic quantum systems. Many numerical methods are available, but typically one simply discretizes Maxwell's equations [9] or expands the solutions in a truncated basis such as planewaves [10] and solves the resulting finite problem.

\subsection{Photonic band gaps in three dimensions}

In one dimension, any periodic structure exhibits a band gap, a fact that was first observed by Lord Rayleigh in 1887 [11], and which appears in nature as the iridescent colors of butterfly wings, abalone 
shells, and certain crystalline minerals. Although this can be seen as a consequence of coherent reflections from interfaces spaced at half-wavelength intervals, such an analysis is not easily generalized to higher dimensions, a difficulty that perhaps explains the 100-year interval between the identification of photonic band gaps in one dimension and those in two or three dimensions. A more general perspective is that the eigenvalues of a Hermitian operator minimize a variational problem, in this case:

$\omega_{n, \mathbf{k}}^{2}=\min _{\mathbf{E}_{n, \mathbf{k}}} \frac{\int\left|(\nabla+i \mathbf{k}) \times \mathbf{E}_{n, \mathbf{k}}\right|^{2}}{\int \varepsilon\left|E_{n, \mathbf{k}}\right|^{2}} c^{2}$,

in terms of the periodic electric field envelope $\mathbf{E}_{n, \mathbf{k}}$, where the numerator minimizes the "kinetic energy" and the denominator minimizes the "potential energy". Here, the higher $(n>1)$ bands are additionally constrained to be orthogonal to the lower bands:

$\int \mathbf{H}_{m, \mathbf{k}}^{*} \cdot \mathbf{H}_{n, \mathbf{k}}=\int \varepsilon \mathbf{E}_{m, \mathbf{k}}^{*} \cdot \mathbf{E}_{n, \mathbf{k}}=0$

for $m<n$. Thus, at each $\mathbf{k}$, there will be a gap between the lower "dielectric" bands concentrated in the high dielectric (low potential) and the upper "air" bands that are less concentrated in the high dielectric: the air bands are forced out by the orthogonality condition, or otherwise must have fast oscillations that increase their kinetic energy. These dielectric/air bands are analogous to the valence/conduction bands in a semiconductor.

The challenge of opening a large gap in three dimensions is increased, however, by the vectorial nature of the fields and their continuity boundary conditions, and turns out to require a structure with high-index contrast (e.g. Si and air), an interwoven network of thin dielectric "veins" along which field lines can run, and (ideally) an fcc (e.g. diamond) crystal structure to most closely approximate a uniform periodicity in all directions. The first example that was identified was, in fact, the diamond lattice of spheres from Fig. 1, by Ho et al. in 1990 [8]. Since then, however, the focus has been on finding structures more amenable to fabrication, and one popular strategy has been layer-by-layer fabrication, in which 2D-patterned layers are etched and stacked by microfabrication methods similar to those developed for integrated circuits [12-15]. One such example is depicted in Fig. 2, comprising an alternating sequence of 2D-periodic arrays of dielectric rods and holes, connecting into a diamond topology [14]; photographs of actual fabricated structures in progress [16] are shown at right. Many other 3D crystal fabrication strategies have also been employed, from colloidal self-assembly [17] to holography [18].

\section{A simple model system: $3 D$ to $2 D$ to $1 D$ to 0D}

An interesting feature of the structure from Fig. 2 is that its layers mimic much simpler two-dimensional photonic crystals, yet it retains a complete band gap in three dimensions: the introduction of a defect in a layer localizes a photon mode that quantitatively resembles that of the corresponding structure in two dimensions [19]. In particular, the analogue of the "rod" layers of the crystal from Fig. 2 is a two-dimensional array of dielectric cylinders arranged in a hexagonal lattice, which has a large band gap for the TM polarization of light: electric field perpendicular to the 2D plane. (In 2D, one can divide photons into two decoupled polarizations, TM and TE.) The resemblance between 3D and 2D is illustrated in Fig. 3, where the modes of equivalent point and line defects are compared in the two systems. Line defects, as in the removed column of rods in Fig. 3 (top), localize light to a line-like "1D" region, forming a waveguide as discussed below. Point defects, as in the single missing rod of Fig. 3 (bottom), localize light in a "OD" region to form a cavity as described below. In both cases, the modes in 3D quantitatively resemble those in 2D, including being over $95 \%$ TM-polarized in the mid-plane, but are confined in the vertical dimension as well (as seen by the cross-section). Because of this capability to mimic two dimensions in three, we will illustrate the capabilities of photonic crystals in the context of those simpler 2D systems. As a model system, we 

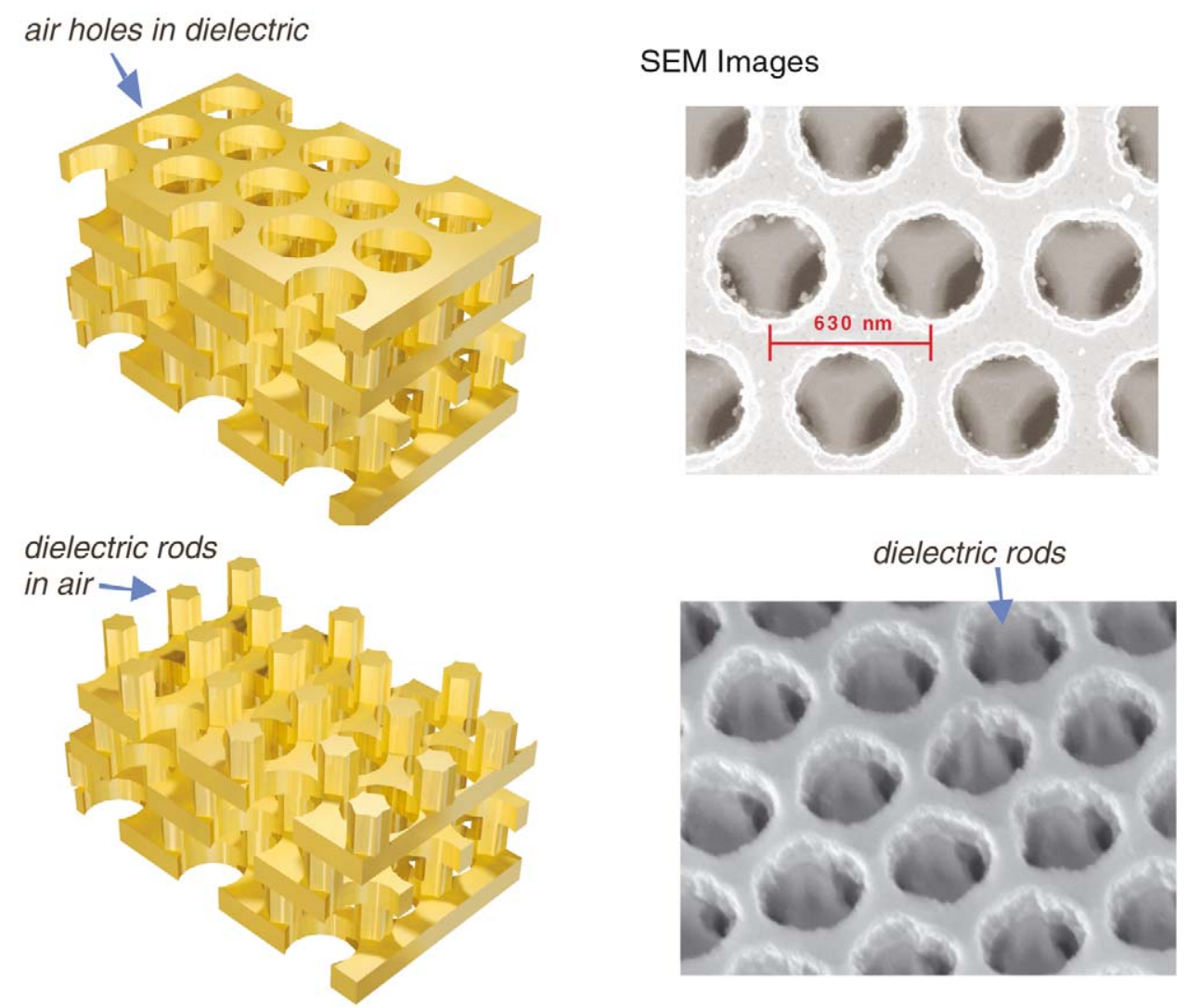

Fig. 2. A layer-by-layer photonic crystal with a complete gap ( $21 \%$ for Si/air), where the layers mimic simpler two-dimensional crystals. (left) Rendered images, with two truncations to show the layers that resemble arrays of air holes and arrays of rods, respectively. (right) SEM of fabricated structure for gap at $\lambda=1.55 \mu \mathrm{m}$.

consider rods with a refractive index of around 3.4 (e.g. GaAs or $\mathrm{Si}$ at the telecommunications wavelength $\lambda=1.55 \mu \mathrm{m}$ ), lattice constant (periodicity) $a$, and radius $0.2 a$, arranged in a square lattice for convenience (the essential physics is unchanged from a hexagonal lattice, and the 3D structure can be designed with a square lattice as well [15]).

By making a linear defect as above, we can support a single photon mode in the band gap whose field is extended along the defect but decays exponentially in the transverse direction (away from the defect). In fact, this mode propagates along the defect, which therefore forms a waveguide. Such a waveguide, however, has many unusual characteristics compared to a conventional dielectric waveguide based on total internal reflection (e.g. optical fiber). For one thing, the light need not be confined in a higher dielectric region, and can even be confined primarily in air, a property that is being exploited to circumvent intrinsic absorption losses of existing materials [20]. Moreover, the band gap forbids light from scattering laterally no matter what happens in the waveguide, so that the photon is effectively trapped in a one-dimensional system. This means, for example, that a sharp ( $\lambda$-scale!) bend in a photonic-crystal waveguide, if it is symmetric and single-mode, can exhibit broad resonances where the transmission approaches $100 \%$, as illustrated in Fig. 4 [21]. This property has been directly observed in experiment [22], and a comparison of measured and simulated transmission (at microwave frequencies) is shown in Fig. 5. The gap's suppression of scattering may even reduce the susceptibility to disorder-induced losses com- 

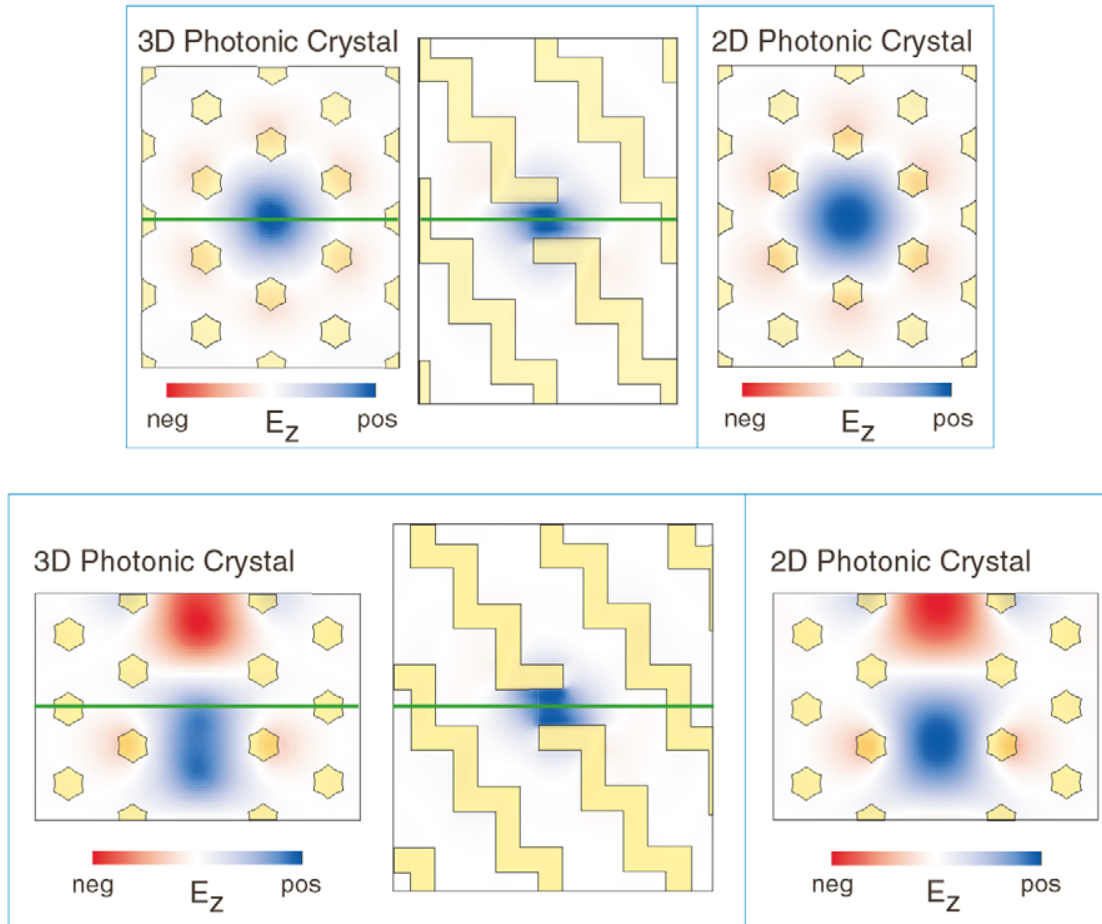

Fig. 3. Comparison of defect modes in a rod layer of the 3D crystal of Fig. 2 and a matching cross-section 2D crystal. (top) Linedefect waveguide (removed column of rods). (bottom) Point defect cavity (removed rod). The $E_{z}$ field component (perpendicular to the $2 \mathrm{D}$ plane) is shown in horizontal and vertical cross-sections, with blue/white/red indicating negative/zero/positive.

pared to a comparable conventional waveguide, eliminating channels of radiation loss without worsening reflection [23]. Finally, at the edge of the Brillouin zone, the group velocity of the waveguided mode drops to zero; this "slow light" capability is also absent in non-periodic index-guided waveguides, and can be used to enhance material properties such as gain or nonlinearity [24].

The second basic defect type is the point-like defect, which can trap a single photon state in the gap whose field decays exponentially in all directions away from the defect (a "zero-dimensional" photon). By changing the defect parameters, one can tune both the frequency and the symmetry of the mode [25], as shown in Fig. 6. For example, by reducing the radius of a single rod (eventually removing it entirely), one can "push up" a single monopole-like state from the lower edge of the gap. Conversely, by increasing the radius of a single rod, one "pulls down" a double-degenerate dipole-like state from the upper edge of the gap.
Further increasing the radius will pull down quadrupole, hexapole, and other higher-order modes. Besides the frequency and symmetry, another critical characteristic of such a cavity mode is the rate at which it leaks out of the cavity, characterized by a dimensionless quality factor $Q$ (roughly, lifetime times frequency). In a photonic crystal, the only mechanism for such leakage is the finite size of the crystal: $Q$ increases exponentially with the number of crystal periods that surround the defect (often by a factor of 10 per period). A unique feature of photonic-crystal cavities, moreover, is that $Q$ is independent of the modal size, which approaches the optimal half-wavelength diameter $(\lambda / 2 n)$. Dielectric microcavities without band gaps, in contrast, are typified by ring resonators (a waveguide bent into a circle), whose radiative losses increase rapidly as the ring diameter is decreased. This combination of high $Q$ and small volume makes photonic-crystal cavities ideal for integrated optical devices: high $Q$ allows narrow-bandwidth filters 


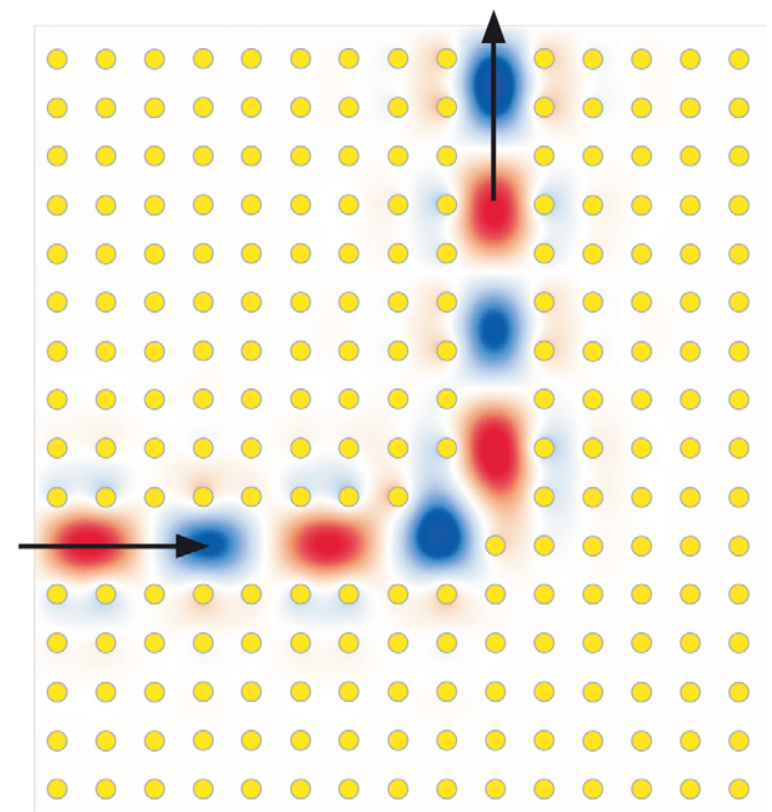

Fig. 4. Resonant $100 \%$ transmission of an air-guided, linedefect waveguide mode around a $90^{\circ}$ bend; such resonances occur whenever one has a complete band gap, single-mode defects, and mirror symmetry in the bend.

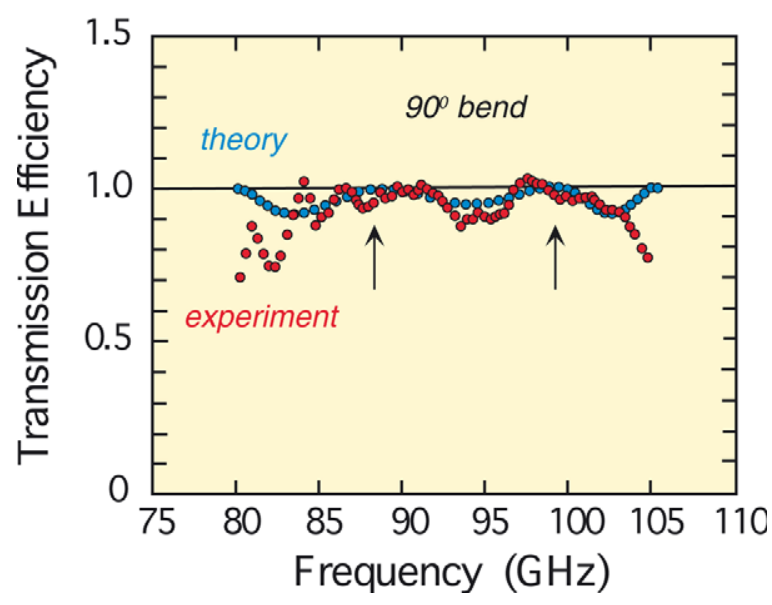

Fig. 5. Experimental vs. theoretical (simulation) transmission around the bend of Fig. 4, constructed with long alumina rods and operated at microwave frequencies.

and strong sensitivity to external tuning, while small volumes increase integration density and heighten the strength of nonlinear effects.

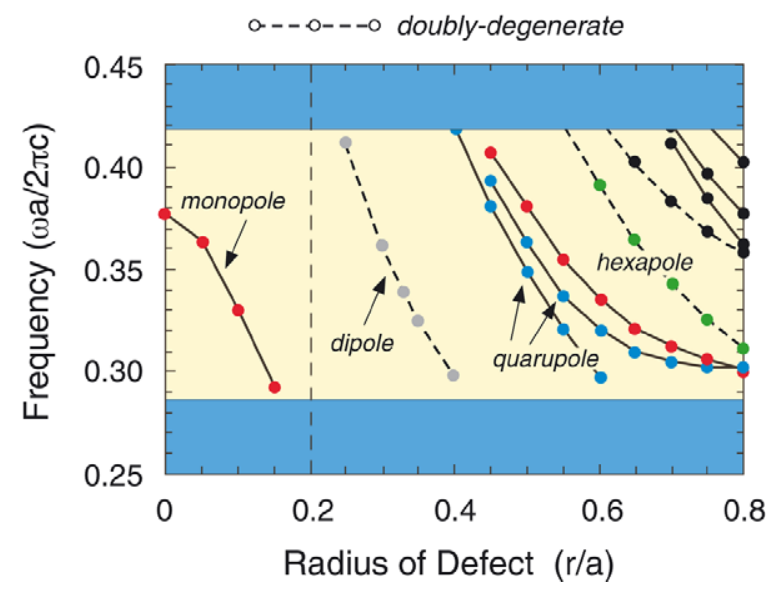

Fig. 6. Frequencies of point defect states, as a function of defect rod radius, as the radius of a single rod is varied up or down from the bulk value of $0.2 a$. Solid lines are singly degenerate and dashed lines are doubly degenerate modes.

\section{Devices by symmetry}

Photonic-crystal waveguides and microcavities are building blocks that can be composed, within the impenetrable surroundings of the photonic band gap, to create a wide variety of different passive and active optical components with potentially near-optimal performance characteristics. Morever, the one-dimensional nature of the waveguides, the arbitrary lifetimes and tunability of the cavities, and the band gap's prohibition of extraneous scattering, enables the analytical design of many devices by a priori principles of symmetry and resonance, with minimal numerical tuning. The bend of Fig. 4 was an example of this: the existence of $100 \%$ transmission resonances is guaranteed by the single-mode and symmetric nature of the bend, which is thereby mathematically equivalent to the simple 1D resonant tunneling problem well known from quantum mechanics [21]. Another way of looking at the bend is to model it as a pair of identical waveguides coupled by a resonant cavity, which is known from coupled-mode theory (based on general principles of energy conservation and time-reversal symmetry) to have $100 \%$ transmission on resonance. This coupled-mode model is only a rough description of the bend, because the bend location is at best a very low- $Q$ res- 
onantor, but this picture is ideally suited to the study of high- $Q$ filters.

In particular, one of the most prominent devices in the telecommunications industry is the "channeldrop" filter, e.g. for use in wavelength-division multiplexing: this device "drops" a single carrier wavelength from one waveguide to another waveguide while other frequencies propagate unaffected in the original guide. Photonic crystals create the opportunity to design such a filter with minimal size and maximal tunability while retaining high $Q$ (narrow bandwidth). An example channel-drop design in our model system is shown in Fig. 7, where it exhibits $100 \%$ dropping efficiency for a single frequency. This design illustrates the basic elements of any channel-drop device: two (typically parallel) waveguides coupled by a resonant cavity, so that the only coupling (dropping) occurs at the resonant frequency of the microcavity. These elements are not sufficient for perfect efficiency, however, because in general the resonant cavity will couple light into four directions: forward and backward in the drop waveguide and some transmission and reflection in the original waveguide. Fan et al. [26], however, have shown that $100 \%$ dropping into a single direction of the drop waveguide, along with zero reflection and transmission in the original waveguide, can be achieved by imposing three simple symmetry conditions on the resonant cavity:
1. The resonator must possess (at least) two resonant modes at (nearly) the same frequency. This condition must be forced by slight design tuning, because the intrinsic symmetry does not support such a degeneracy.

2. There must be two mirror symmetry planes bisecting the cavity, one perpendicular and one parallel to the waveguides. The two resonant modes must be even and odd, respectively, under the mirror plane perpendicular to the waveguides (this parity can occur intrinsically and need not be forced).

3. The modes must have (nearly) equal $Q$.

The design of Fig. 7 satisfies all these conditions, which are both necessary and sufficient (if the waveguides are single-mode), where the two resonant modes come from the doubly degenerate hexapole modes of an isolated enlarged-radius point defect-these modes satisfy the requisite symmetries, and the degeneracy is broken by the waveguides but is restored (forced) by tuning an adjacent rod radius. The general design principles can be understood as follows. Because the modes have equal $Q$ and frequency, the input waveguide couples equally to both (at resonance). When the modes decay back into the waveguides, however, their opposite parity from condition (2) means that the exiting fields cancel in the reflected direction and in one of the drop directions. The only remain-

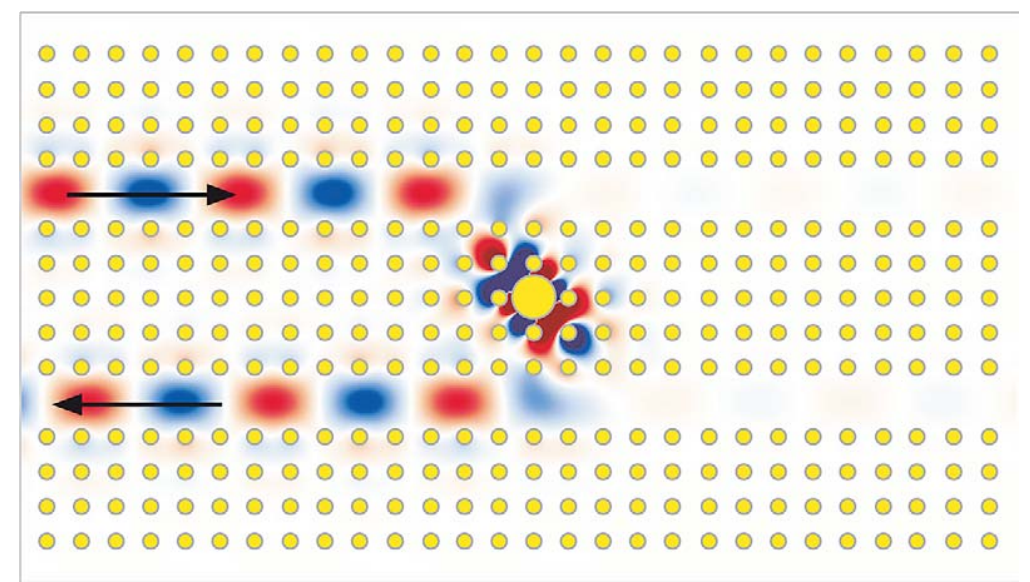

Fig. 7. Channel-drop filter coupling two line-defect waveguides at a single resonant frequency, via a doubly degenerate hexapole defect (center) that obeys the necessary symmetry conditions for $100 \%$ transfer efficiency. 
ing decay channels are the transmitted and drop directions, from which follows the $100 \%$ resonant transmission that is always obtained in two-channel coupled-mode problems.

Other passive optical devices can be similarly designed. For example, two waveguides can be crossed with arbitrarily low crosstalk by mediating the crossing with a doubly degenerate cavity whose symmetry only permits coupling to opposite and not adjacent waveguide ports [27]; this idea is exploited in Fig. 8 (top). A two-way Y-junction splitter $(50 \%$ transmission to each output waveguide) can be designed by realizing that it is equivalent to the reflection-free two-port coupling problem when the total coupling (decay rate of junction resonance) to the two output ports is equal to the coupling back to the input [28]. A consequence of this is the theorem, well-known from microwave scattering, that a junction coupling equally to all three outputs will necessarily have reflections - to eliminate reflection, one must paradoxically introduce obstructions that reduce coupling to the output ports; this has been done in Fig. 8 (bottom) by adding small rods partially blocking the two outputs.

Such design principles, moreover, are not limited to passive devices, but extend also to active and nonlinear components. For example, consider the case of an input line-defect waveguide coupled to an output waveguide via a resonant cavity, which will have a resonance of $100 \%$ transmission by the general principles above (thanks to the photonic crystals' prohibition of radiative loss), as shown in Fig. 9 (top). Now, recall that the materials of the cavity will probably have some Kerr nonlinearity, giving a field-dependent index shift $\Delta n \sim|\mathbf{E}|^{2}$. If we operate this device at a frequency $\omega_{0}$ below the resonance, we will initially have low transmission, but as we increase the power the resonant frequency will be shifted down
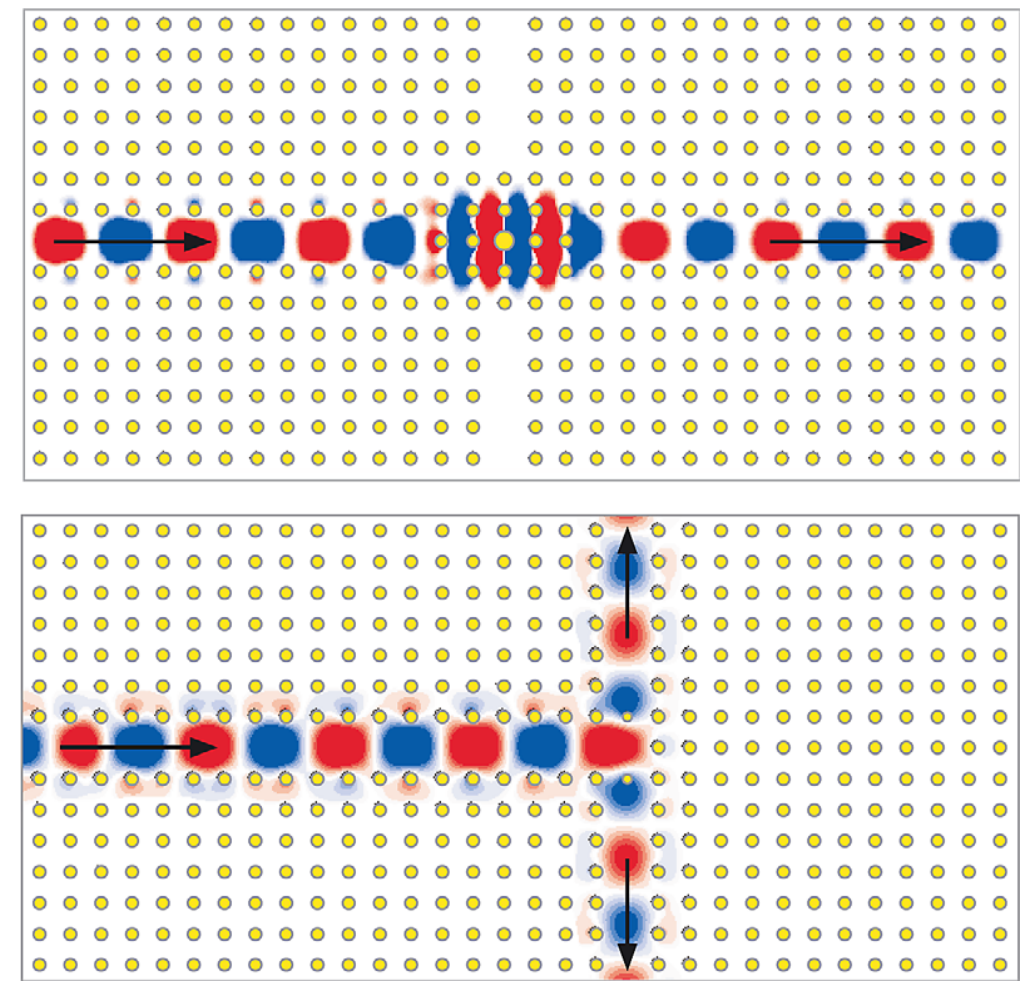

Fig. 8. Devices designed with simple symmetry and resonance principles. (top) Intersection of two photonic-crystal waveguides with only $10^{-4}(40 \mathrm{~dB})$ crosstalk, mediated by a doubly degenerate dipole-like mode at the intersection. (bottom) Perfect $3 \mathrm{~dB} \mathrm{Y}$ splitter, with reflections prevented by two small rods partially blocking the outputs in order to satisfy the resonance condition. 

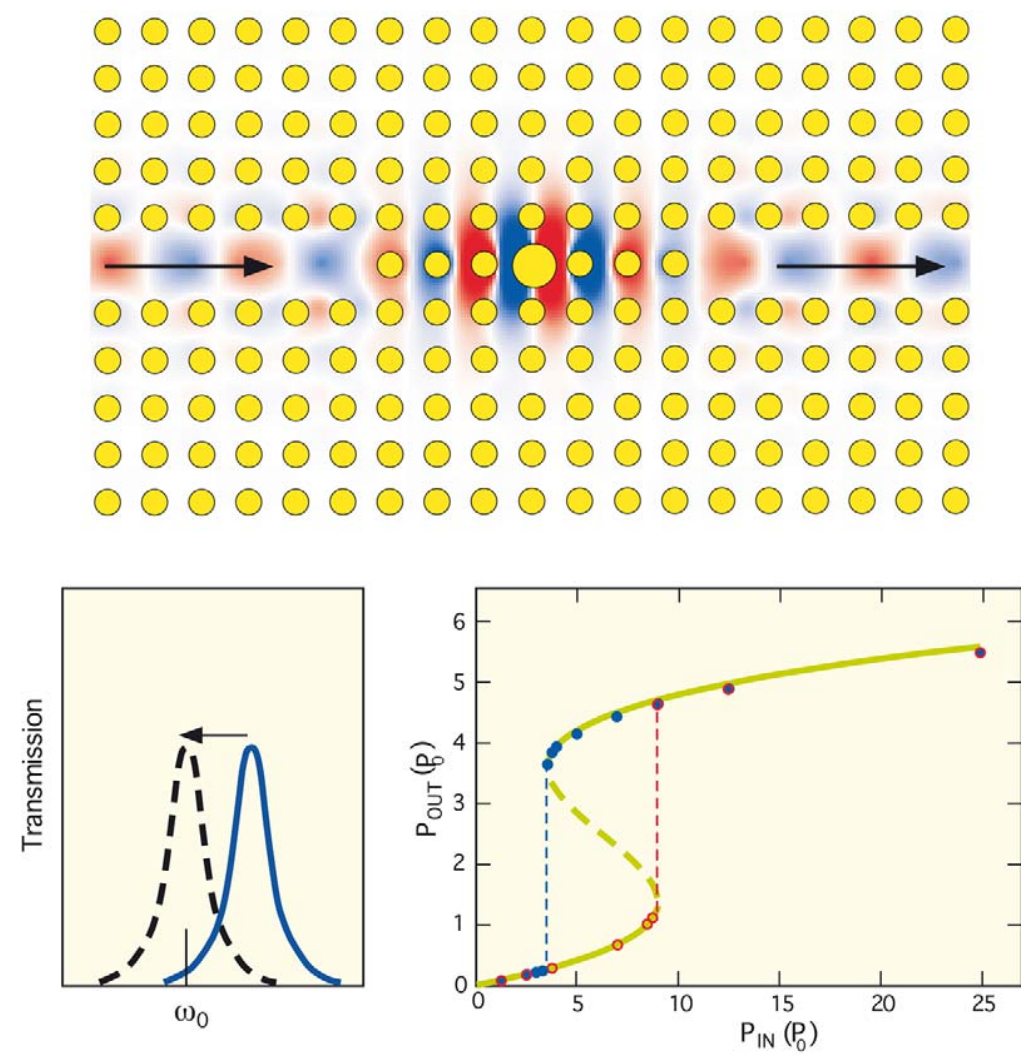

Fig. 9. (top) Resonant $100 \%$ transmission between two waveguides coupled by a resonant cavity. (bottom-left) Lorentzian transmission spectrum, shifted (dashed line) to lower frequency by a Kerr nonlinearity as power increases. (bottom-right) Output vs. input power (arbitrary units) of nonlinear resonant cavity, showing bistable hysteresis response. Dots are full simulations, line is semianalytical perturbation-theory prediction [29] (dashed line is unstable solution).

until transmission shoots up to $100 \%$. Moreover, there is a nonlinear feedback: as we shift into the resonance, more power couples into the cavity and the nonlinear shift increases (Fig. 9, bottom-left); conversely, as we further increase the power and shift out of the resonance, less power couples into the cavity and the nonlinear shift decreases. This feedback produces a hysteresis curve in the output vs. input power, as shown in Fig. 9 (bottom-right). Such behavior, called optical bistability, creates a kind of optical transistor, with a correspondingly wide range of applications from optical logic to rectification to amplification to switching. Although the bistability effect is well-known, photonic crystals promise its most optimal implementation: the threshold power for the onset of bistability is proportional to $V / Q^{2}$, where $V$ is the modal volume, so the ability of photonic band gaps to combine small $V$ with high $Q$ is critical. Thresholds of a few milliwatts are predicted for Si photonic crystals [29].

\section{Going without a complete gap}

Although photonic crystals with complete (omnidirectional) band gaps are the most ideal and simple from a theoretical point of view, and great progress has been made in their experimental realization, the fabrication of three-dimensionally periodic structures remains challenging. As a nearer-term alternative, researchers have explored alternative, "hybrid" structures that combine incomplete photonic band gaps with either conven- 
tional index guiding (photonic-crystal slabs) or translational/axial symmetry (photonic band-gap fibers).

Photonic-crystal slabs are two-dimensionally periodic dielectric structures with (typically) constant cross-section and finite thickness, which combine an in-plane band gap with vertical confinement via index guiding (the analogue of traditional total internal reflection) [2]. These slabs can come in two basic topologies, shown in Fig. 10: (a) dielectric rods in air and (b) air cylinders/holes in dielectric. The materials above and below the slab have a much lower average index to facilitate index guiding; typically, the slab is $\mathrm{Si}$ or GaAs and rests on oxide, or can even be a suspended air membrane (in the case of the hole slab). Such structures can still support leakage-free waveguides confined in the plane by the band gap, although they cannot guide light primarily in air because of the constraint of vertical index guiding. Because these structures do not have a complete photonic band gap, however, radiation losses are inevitable when translational symmetry is broken (e.g. by a cavity or a waveguide bend), complicating the design of efficient devices. Nevertheless, if vertical leakage from a cavity can be made sufficiently small (many times smaller than the leakage into desired outlets (a) Dielectric rods in air

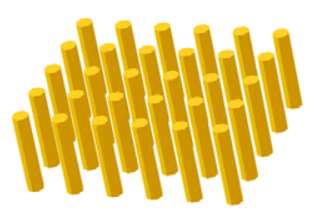

(b) Air cylinders in dielectric

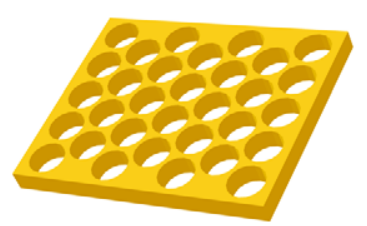

(c) Bragg/Omniguide fiber

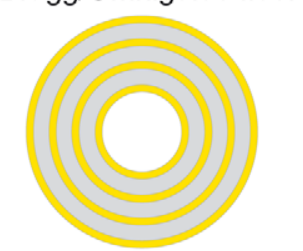

(d) Photonic-crystal fiber

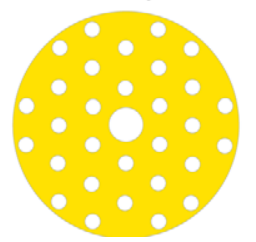

Fig. 10. Schematics of hybrid structures, which combine an incomplete 1D/2D gap with: (a, b) vertical index guiding (photonic-crystal slabs), via a high-index 2D periodic structure of finite height; (c, d) axial symmetry (photonic band-gap fibers). such as adjacent waveguides), losses can be acceptable; several strategies for minimizing this vertical leakage exist [2], and experimental slab cavities have been fabricated with lifetimes of thousands of optical periods [30,31].

Another promising hybrid structure is that of photonic band-gap fibers, which have a translational symmetry along the fiber axis that allows light to be confined within the fiber by the band gap of a 1D- or 2D-periodic crystal. The 1D-periodic case, formed by periodic concentric rings as in Fig. 10(c), is called a Bragg fiber and dates back to work by Yeh et al. in 1978 [32], with a more recent twist, called an Omniguide fiber [20,33], of using omnidirectional reflectors [34] for the mirrors. The other alternative is a 2D-periodic crystal as in Fig. 10(d), typically formed by air veins in silica, called a photonic-crystal fiber and introduced by Knight et al. in 1998 [35]. In both cases, the translational symmetry means that there is a conserved wavenumber $k$ (a special case of the Bloch wavevector) along the fiber axis, and at a given $k$ there is a range of band-gap frequencies where light cannot propagate in the crystal. This can be used to trap light in a central cavity/defect, like those in Fig. 10, and can even guide light primarily in air like a complete band gap. Such airguidance has the potential to circumvent the intrinsic material absorption and nonlinearity that currently limits fiber performance, and waveguided modes have been experimentally demonstrated with 4-5 orders of magnitude lower losses than those of the constituent solid materials [20].

\section{Concluding remarks}

Ever-increasing bandwidth demands have driven more and more applications to ride on optical carrier frequency, with a corresponding demand for maximally miniaturized, solid-state optical devices. This has been especially true in the world of digital telecommunications, where the goal is to eventually integrate many such processing devices on a single chip. To achieve such localization, however, it will be necessary to exploit mechanisms that go beyond index guidance (total internal reflection), and photonic crystals thus pro- 
vide a new and promising foundation upon which to build future optical devices. The building blocks of such devices are waveguides and microcavities, and in both cases photonic crystals promise important advantages in localization, tunability, and efficiency. Moreover, the ability of photonic crystals to control light in new ways, to alter both its localization and its propagation characteristics, has the potential to transform many other fields of optical technology. Perhaps chief among these fields is that of the optical fiber, where band-gap fibers have the ability to dramatically alter the landscape of material-limited loss, nonlinearity, and other properties.

\section{Acknowledgments}

This work was supported in part by the Materials Research Science and Engineering Center program of the National Science Foundation under award DMR-9400334. We are also especially indebted to M.L. Povinelli, Minghao Qi, Attila Mekis, and Shanhui Fan for sharing the fruits of their efforts.

\section{References}

[1] Joannopoulos JD, Meade RD, Winn JN. Photonic crystals: molding the flow of light. Princeton University Press, 1995.

[2] Johnson SG, Joannopoulos JD. Photonic crystals: the road from theory to practice. Boston: Kluwer, 2002.

[3] Yablonovitch E. Inhibited spontaneous emission in solidstate physics and electronics. Phys Rev Lett 1987;58:2059-62.

[4] John S. Strong localization of photons in certain disordered dielectric superlattices. Phys Rev Lett 1987;58:2486-9.

[5] Bloch F. Über die Quantenmechanik der Electronen in Kristallgittern. Z Phys 1928;52:555-600.

[6] Floquet G. Sur les équations différentielles linéaries à coefficients périodiques. Ann École Norm Sup 1883;12:47-88.

[7] Ashcroft NW, Mermin ND. Solid state physics. Philadelphia: Holt Saunders, 1976.

[8] Ho KM, Chan CT, Soukoulis CM. Existence of a photonic gap in periodic dielectric structures. Phys Rev Lett 1990;65:3152-5.

[9] Kunz KS, Luebbers RJ. The finite-difference time-domain method for electromagnetics. Boca Raton: CRC Press, 1993.
[10] Johnson SG, Joannopoulos JD. Block-iterative frequencydomain methods for Maxwell's equations in a planewave basis. Opt Express 2001;8(3):173-90 [URL: http://www.opticsexpress.org/abstract.cfm?URI=OPEX-83-173].

[11] Lord Rayleigh. On the maintenance of vibrations by forces of double frequency, and on the propagation of waves through a medium endowed with a periodic structure. Philos Mag 1887;24:145-59.

[12] Ho KM, Chan CT, Soukoulis CM, Biswas R, Sigalas M. Photonic band gaps in three dimensions: new layer-bylayer periodic structures. Solid State Commun 1994;89:413-6.

[13] Sözüer HS, Dowling JP. Photonic band calculations for woodpile structures. J Mod Opt 1994;41(2):231-9.

[14] Johnson SG, Joannopoulos JD. Three-dimensionally periodic dielectric layered structure with omnidirectional photonic band gap. Appl Phys Lett 2000;77:3490-2.

[15] Roundy D, Joannopoulos JD. Photonic crystal structure with square symmetry within each layer and a threedimensional band gap. Appl Phys Lett 2003;82(22):3835-7.

[16] Qi M, Smith H. Private communications, 2003.

[17] Vlasov YA, Bo X-Z, Sturm JC, Norris DJ. On-chip natural assembly of silicon photonic bandgap crystals. Nature 2001;414:289-93.

[18] Sharp DN, Campbell M, Dedman ER, Harrison MT, Denning RG, Turberfield AJ. Photonic crystals for the visible spectrum by holographic lithography. Optical Quantum Electron 2002;34:3-12.

[19] Povinelli ML, Johnson SG, Fan S, Joannopoulos JD. Emulation of two-dimensional photonic crystal defect modes in a photonic crystal with a three-dimensional photonic band gap. Phys Rev B 2001;64:075313.

[20] Temelkuran B, Hart SD, Benoit G, Joannopoulos JD, Fink Y. Wavelength-scalable hollow optical fibres with large photonic bandgaps for $\mathrm{CO}_{2}$ laser transmission. Nature 2002;420:650-3.

[21] Mekis A, Chen JC, Kurland I, Fan S, Villeneuve PR, Joannopoulos JD. High transmission through sharp bends in photonic crystal waveguides. Phys Rev Lett 1996;77(18):3787-90.

[22] Lin S-Y, Chow E, Hietala V, Villeneuve PR, Joannopoulos JD. Experimental demonstration of guiding and bending of electromagnetic waves in a photonic crystal. Science 1998;282:274-6.

[23] Povinelli ML, Johnson SG, Lidorikis E, Joannopoulos JD, Soljačić M. Reduction of disorder-induced scattering losses using photonic-crystal waveguides [submitted for publication].

[24] Soljačić M, Johnson SG, Fan S, Ibanescu M, Ippen E, Joannopoulos JD. Photonic-crystal slow-light enhancement of non-linear phase sensitivity. J Opt Soc Am B 2002;19:2052-9.

[25] Villeneuve PR, Fan S, Joannopoulos JD. Microcavities in photonic crystals: mode symmetry, tunability, and coupling efficiency. Phys Rev B 1996;54:7837-42. 
[26] Fan S, Villeneuve PR, Joannopoulos JD, Haus HA. Channel drop tunneling through localized states. Phys Rev Lett 1998;80(5):960-3.

[27] Johnson SG, Manolatau C, Fan S, Villeneuve PR, Joannopoulos JD. Elimination of cross talk in waveguide intersections. Opt Lett 1998;23(23):1855-7.

[28] Fan S, Johnson SG, Joannopoulos JD, Manolatou C, Haus HA. Waveguide branches in photonic crystals. J Opt Soc Am B 2001;18(2):162-5.

[29] Soljačić M, Ibanescu M, Johnson SG, Fink Y, Joannopoulos JD. Optimal bistable switching in non-linear photonic crystals. Phys Rev E Rapid Commun 2002;66:055601.

[30] Lin SY, Chow E, Johnson SG, Joannopoulos JD. Direct measurement of the quality factor in a two-dimensional photonic-crystal microcavity. Opt Lett 2001;26(23):1903-5.
[31] Lončar M, Yoshie T, Scherer A, Gogna P, Qiu Y. Lowthreshold photonic crystal laser. Appl Phys Lett 2002;81(15):2680-2.

[32] Yeh P, Yariv A, Marom E. Theory of Bragg fiber. J Opt Soc Am 1978;68:1196-201.

[33] Johnson SG, Ibanescu M, Skorobogatiy M, Weisberg O, Engeness TD, Soljačić M et al. Low-loss asymptotically single-mode propagation in large-core OmniGuide fibers. Opt Express 2001;9(13):748-79 [URL: http://www.opticsexpress.org/abstract.cfm?URI=OPEX-913-748].

[34] Fink Y, Winn JN, Fan S, Chen C, Michel J, Joannopoulos JD et al. A dielectric omnidirectional reflector. Science 1998;282:1679-82.

[35] Knight JC, Broeng J, Birks TA, Russell PS-J. Photonic band gap guidance in optical fibers. Science 1998;282:1476-8. 